\title{
Quantitative association between nocturnal voiding frequency and objective sleep quality in the general elderly population: the Heijō-kyō cohort
}

\section{Kenji Obayashi ${ }^{\mathrm{a}, *}$, Keigo Saeki ${ }^{\mathrm{a}}$, Norio Kurumatani ${ }^{\mathrm{a}}$}

aDepartment of Community Health and Epidemiology, Nara Medical University School of Medicine, Nara, Japan

*Corresponding author. Department of Community Health and Epidemiology, Nara Medical University School of Medicine, 840 Shijocho, Kashiharashi, Nara, 634-8521, Japan. Tel.: +81-744-22-3051; fax: +81-744-25-7657.

E-mail address: obayashi@naramed-u.ac.jp (Kenji Obayashi) 


\section{Abstract}

Objective: A significant association between nocturia and subjective sleep quality has previously been reported; however, the association between nocturia and objective sleep quality remains unclear. The purpose of this study was to evaluate the quantitative association between nocturnal voiding (NV) frequency and objective sleep quality in a large, general, elderly population.

Methods: Nocturnal voiding frequency, objective sleep quality, and subjective sleep quality were measured among 1086 community-based elderly individuals using actigraphy and the Pittsburgh Sleep Quality Index (PSQI) questionnaire.

Results: In multivariate analyses adjusted for potential confounding factors (such as age, gender, body mass index, medication use, renal function, bedtime, rising time, daytime physical activity, endogenous melatonin levels, and bedroom light levels), increased NV frequency, ranging from zero, one, two, three or more voids, was significantly associated with poorer objective sleep quality, including lower sleep efficiency (SE) and longer wake after sleep onset (WASO) (mean SE, 86.3, 84.8, 83.6, and 81.2\%, respectively; $p$ for trend $<0.001$; mean WASO: 42.6, 49.0, 53.6, and $66.1 \mathrm{~min}$, respectively; $p$ for trend $<0.001$ ), but shorter sleep onset latency (SOL) (mean SOL, 3.0, 3.0, 2.8, and $2.8 \log$ min, respectively; $p$ for trend $=0.018)$. In addition, an increased NV frequency was significantly associated with poorer subjective sleep quality in a multivariate model (mean PSQI global score, 4.60, 4.86, 5.22, and 5.48, respectively; $p$ for trend 0.012 ).

Conclusion: The present study revealed a quantitative association between NV frequency and objective sleep quality in the general elderly population. 


\section{Keywords:}

Nocturia

Nocturnal voiding frequency

Sleep quality

Actigraphy

Melatonin 


\section{Introduction}

Previous epidemiological studies have revealed that the prevalence of self-reported sleep problems increases with age, and that up to $40 \%$ of elderly individuals suffer from problems related to the initiation and maintenance of sleep [1-4]. Sleep problems have commonly been associated with an increased risk of depression, dementia, cardiovascular diseases, and mortality [5-8]. Therefore, sleep problems in elderly individuals are important public health issues.

Nocturnal voiding (NV) frequency increases with age, and $>20 \%$ of elderly individuals have two or more nocturnal voids $[9,10]$. An increased frequency of nocturnal voiding is a risk factor for both bone fractures and cardiovascular disease $[11,12]$. Although the International Continence Society defines nocturia as a condition in which the affected individual wakes one or more times at night to void, it remains unclear as to whether there is a quantitative association between NV frequency and pathophysiological significance [13].

Several previous studies have reported a significant association between nocturia and sleep quality; however, most of these studies characterized nocturia using a cutoff point of NV frequency, and sleep quality was assessed using self-reported questionnaires [14-18]. Although some inconsistency between subjective and objective sleep measures has been reported, the association between nocturia and objective sleep quality remains unclear [19]. A recent study suggested that there is a quantitative association between NV frequency, ranging from zero to four or more, and sleep quality measured actigraphically; however, the study was limited to generalizability of the findings because it included a small sample size $(n=60)$ and targeted a population with insomnia [20]. Therefore, the quantitative association between $\mathrm{NV}$ frequency and objective sleep quality should be evaluated in a large, general, elderly population. 
In this cross-sectional study of 1086 community-dwelling elderly individuals, the association of continuous and categorical data on NV frequency with objective and subjective sleep quality using actigraphy and Pittsburgh Sleep Quality Index (PSQI) questionnaires was investigated. 


\section{Participants and Methods}

\section{Participants and study protocol}

A total of 1127 community-based elderly individuals aged $\geq 60$ years were voluntarily enrolled into the study Housing environments and health investigation among Japanese older people in Nara, Kansai Region: a prospective community-based cohort (HEIJO-KYO) study between September 2010 and March 2014. Among these, 1086 participants met the inclusion criterion of having completed sleep quality and NV frequency measurements. All participants provided written, informed consent to participate in the study. The Nara Medical University Ethics Committee approved the study protocol.

The study protocols were previously described [21]. Briefly: demographic and medical information were collected using a standardized questionnaire, and measurements (including actigraphic parameters) were taken on two consecutive days. The participants were then instructed to collect urine samples the following night, maintain standardized sleep and urination diaries, and not to consume any alcohol during the monitoring period.

\section{Measuring objective sleep quality}

An actigraph (Actiwatch 2; Respironics Inc., PA, USA), worn on the non-dominant wrist, was used to measure objective sleep quality at 1-min intervals on two consecutive nights. The asleep/awake status at each epoch, sleep onset, and sleep offset were detected automatically using Actiware version 5.5 (Respironics) using the default algorithm [22]. Epochs with activity counts higher than a moderate threshold (40 counts/min) were treated as being awake. Sleep onset was defined as the first minute that was followed by a 10-min immobility period that contained not more than one epoch with any motion count. Sleep offset was defined as the last minute following a 10-min immobility period. 
Objective data (awake/asleep status and sleep onset/offset) and self-reported data (bedtime and rising time) were used to calculate four actigraphic sleep parameters each night: (1) total sleep time (TST), the total time spent asleep (below the activity threshold of 40 counts/min) between sleep onset and offset; (2) sleep efficiency (SE), the \%TST divided by the time between bedtime and rising time, as derived from the self-reported sleep diary; (3) wake after sleep onset (WASO), the time spent awake (above the activity threshold of 40 counts/min) between the sleep onset and rising times; and (4) sleep onset latency (SOL), the time between bedtime and sleep onset. Probable sleep-disordered breathing (SDB) was defined as an $\mathrm{SE}<70 \%$ and/or a TST $<5 \mathrm{~h}$ because odds ratio for SDB was significantly higher in the elderly population with this criteria [23].

\section{Measuring subjective sleep quality}

Subjective sleep quality was measured using PSQI questionnaires. Specifically, sleep quality over the previous month was determined using seven subscales that measure different components of sleep: sleep quality, latency, duration, efficiency, disturbance, sleep

medication use, and daytime dysfunction. Each component was scored from 0-3, with 3 indicating the worst quality of sleep [24].

\section{Measuring nocturnal voiding frequency}

Nocturnal voiding frequency, except for the last void at bedtime and the first void in the morning, was logged using a standardized urination diary. In the first 189 participants, the correlation of NV frequency over 4 months was assessed. The agreement for two or more NVs between the two data sets was moderate (Kappa coefficient $=0.55)$. 


\section{Other measurements}

Body mass index (BMI) was calculated as weight divided by the square of height in meters $\left(\mathrm{kg} / \mathrm{m}^{2}\right)$. The use of antihypertensive drugs and sleep medications, and benign prostatic hyperplasia $(\mathrm{BPH})$ were evaluated using a questionnaire. Diabetes mellitus was defined according to: medical history, the current use of antidiabetic agents, fasting plasma glucose levels, and plasma glycated hemoglobin levels. The estimated glomerular filtration rate (eGFR) was calculated using the formula described by the Japanese Society of Nephrology,

Chronic Kidney Disease Practice Guide. Daytime physical activity was calculated as the mean physical activity over the 2 days, which was evaluated using an actigraph (Actiwatch 2; Respironics Inc., PA, USA) from rising time to bedtime. Endogenous melatonin levels were estimated according to nocturnal urinary 6-sulfatoxymelatonin excretion (UME) using a highly sensitive enzyme-linked immunosorbent assay (ELISA) kit (RE54031; IBL International, Hamburg, Germany) as previously described [21]. Bedroom light intensity was measured at 1-min intervals over two consecutive nights using a portable light meter (LX-28SD; Sato Shouji Inc., Kanagawa, Japan); the mean light intensity from bedtime to rising time was used to define the bedroom light intensity.

\section{Statistical analyses}

Age, gender, BMI, antihypertensive drugs and sleep medication use, diabetes, eGFR, bedtime, rising time, daytime physical activity, UME, and bedroom light intensity were included as independent variables. The mean values for daytime physical activity, bedtime, rising time, bedroom light intensity, and actigraphic sleep parameters on two consecutive days were used for analyses. The UME and SOL had a skewed distribution, and therefore, they were naturally log-transformed for analyses. Trends in the association between NV frequency and the independent variables and sleep parameters were evaluated using linear or logistic 
regression models when variables were normally distributed or categorical data, respectively. Jonckheere-Terpstra tests for trends were used when variables were distributed asymmetrically. Independent variables were adjusted in multivariate models to assess the association between $\mathrm{NV}$ frequency and sleep quality using analysis of covariance (ANCOVA) where there was no serious multicollinearity (all variance inflation factors $<10$ ). Antihypertensive drug use, which was significantly associated with NV frequency, was analyzed as a covariate in multivariate analyses. Sleep medication use was excluded from covariates in the multivariate analysis of subjective sleep measures because information regarding the use of sleep medication was included in the PSQI. Statistical analyses were performed using SPSS version 19.0 for Windows (IBM SPSS Inc., IL, USA). A two-tailed $p$-value of $<0.05$ was considered to be statistically significant. 


\section{Results}

The mean age of the study participants was $71.8 \pm 7.1$ (standard deviation, SD) years, and 511 (47.1\%) were male. Of the 1086 participants, 302 had zero NV, 462 had one NV, 232 had two NVs, and 90 had three or more NVs. Older age, male gender, calcium channel blocker (CCB) and sleep medication use, lower eGFR, earlier bedtime, later rising time, and lower daytime physical activity were significantly associated with increased NV frequency (Table 1). Probable SDB $(n=66)$ did not differ significantly according to NV frequency $\left(p_{\text {trend }}=0.11\right)$.

An increased NV frequency was significantly associated with lower SE, longer WASO, and longer TST $\left(p_{\text {trend }}<0.001\right.$, Table 2$)$. In multivariate analyses adjusted for potential confounders, an increase in NV frequency, ranging from zero to one, two, and three or more voids, was significantly associated with lower SE and longer WASO (mean SE [95\% confidence interval, CI] 86.3\% [85.5-87.2], 84.8\% [84.2-85.5], 83.6\% [82.6-84.6], and 81.2\% [79.6-82.7], respectively, $p_{\text {trend }}<0.001$; mean WASO [95\% CI] $42.6 \mathrm{~min}$ [39.6-45.6], $48.9 \mathrm{~min}$ [46.6-51.3], 53.6 $\min [50.3-57.0]$, and 66.1 $\min$ [60.7-71.5], respectively, $\left.p_{\text {trend }}<0.001\right)$. These significant association trends of NV frequency with SE and WASO were consistent both in the population excluding probable SDB (mean SE $p_{\text {trend }}<0.001$; mean WASO $p_{\text {trend }}<0.001$ ) and in the male population without BPH (mean SE $p_{\text {trend }}=0.005$; mean WASO $p_{\text {trend }}=0.008$ ). Nocturnal voiding frequency was not associated with TST, but was significantly associated with shorter SOL [mean log-transformed SOL (95\%CI) $3.0 \log \min (2.9-3.1), 3.0 \log \min$ (2.9-3.1), $2.8 \log \min (2.7-2.9)$, and $2.8 \log \min (2.6-3.0)$, respectively, $\left.p_{\text {trend }}=0.018\right]$. Compared with the no NV group, all of the other NV groups had a significantly lower SE and longer WASO (mean SE difference [95\%CI] 1.5\% [0.4-2.6], $p=0.006 ; 2.7$ [1.4-4.1], $p<0.001$;

$5.1 \%[3.3-7.0], p<0.001$; mean WASO difference $[95 \% \mathrm{CI}] 6.3 \min [2.5-10.1], p=0.001 ; 11.0$ $\min [6.4-15.7], p<0.001 ; 23.5 \min [17.1-29.9], p<0.001$, respectively).

Regarding the association between NV frequency and subjective sleep quality, an 
increase in NV frequency was associated with higher global PSQI score significantly $\left(p_{\text {trend }}<0.001\right.$, Table 3$)$. Analyses assessing the association between NV frequency and PSQI components indicated that an increase in NV frequency was significantly associated with poorer sleep quality $\left(p_{\text {trend }}<0.001\right)$, prolonged sleep latency $\left(p_{\text {trend }}<0.001\right)$, longer sleep duration $\left(p_{\text {trend }}<0.001\right)$, lower sleep efficiency $\left(p_{\text {trend }}<0.001\right)$, sleep disturbances $\left(p_{\text {trend }}<0.001\right)$, more sleep medication use $\left(p_{\text {trend }}=0.031\right)$, and daytime dysfunction $\left(p_{\text {trend }}=0.039\right)$. In multivariate analyses adjusted for potential confounders such as age, gender, BMI, CCB use, diabetes, eGFR, bedtime, rising time, daytime physical activity, UME, and bedroom light intensity, an increase in NV frequency (zero, one, two, and three or more voids) was significantly associated with higher global PSQI scores (mean global PSQI score [95\%CI] 4.61 [4.23-4.99], 4.87 [4.58-5.16], 5.22 [4.80-5.64], and 5.48 [4.80-6.17], respectively, $\left.p_{\text {trend }}=0.012\right)$ and higher PSQI component scores regarding sleep quality $\left(p_{\text {trend }}<0.001\right)$, sleep latency $\left(p_{\text {trend }}=0.045\right)$, sleep disturbances $\left(p_{\text {trend }}<0.001\right)$, and daytime dysfunction $\left(p_{\text {trend }}=0.005\right)$. However, there was no significant association with sleep duration, sleep efficiency, and sleep medication. Compared with the zero NV group, individuals in the two and three or more NV groups had significantly higher global PSQI scores (adjusted mean difference [95\%CI] $0.61[0.03-1.20], p=0.041$; and 0.87 [0.07-1.68], $p=0.034$, respectively). However, the global PSQI score in the one NV group was comparable to that in the no NV group $(p=0.28)$.

In addition, compared with the less than two NV group, individuals with two or more NV had a significantly lower SE (85.4\% [84.8-85.9] vs 83.0\% [82.2-83.9], $p<0.001)$, longer WASO (46.7 $\min [44.8-48.5]$ vs $56.6 \min [53.7-59.6], \quad p<0.001)$, shorter $\log$-transformed SOL (3.0 log $\min [2.9-3.1]$ vs $2.8 \log \min [2.7-2.9], p=0.009)$, and lower global PSQI score (4.77 [4.54-5.00] vs 5.27 [4.91-5.64], $p=0.028)$ (Table 4). A 0.2 log min shorter log-transformed SOL corresponded to a $1.2 \mathrm{~min}$ shorter SOL. 


\section{Discussion}

The present study demonstrated that NV frequency is significantly associated with objective sleep quality, as evaluated using quantitative analyses regarding the association between NV frequency and actigraphic sleep quality in the general elderly population. Most previous studies have reported an association between nocturia that was categorized using cutoff points for NV frequency and subjective sleep quality. In the present study, NV frequency was treated as continuous data (zero, one, two, and three or more voids per night), and sleep quality was evaluated using actigraphy and self-reported questionnaires.

The associations between objective and subjective measures of sleep quality and NV frequency differed in the present study. Regarding subjective sleep quality, a significant trend toward an association with NV frequency was observed, although no significant difference was observed in the global PSQI score between the one and zero NV categories. In contrast, significant differences were found in the actigraphic SE and WASO between the one and zero NV categories for objective sleep quality. The International Continence Society defines nocturia as a condition where an individual has to wake at night to void one or more times; however, this criterion is controversial because one void per night is considered to be normal by several researchers [25]. The present study suggested that one NV might be related to impaired objective sleep quality, although this might be because statistical power of objective measures is stronger than that of subjective measures because of a smaller variation in objective measures. In addition, some PSQI component measures, such as sleep efficiency and sleep medication, did not differ significantly among the NV frequency groups, possibly because of the relatively large variation. Indeed, a previous study with a large sample size indicated that the possible risk of one NV was related to impaired subjective sleep quality [18]. Another explanation for the stronger statistical power of objective sleep measures compared with subjective ones is that the subjective measures are derived from sleep quality 
over the previous month rather than sleep quality on the day when NV frequency was measured.

It is believed that the present study presents the first evidence suggesting an association between NV frequency and objective sleep quality in a general elderly population. A previous study revealed a quantitative association between NV frequency (zero, one, two, three, and four or more voids per night) and actigraphic sleep quality in 60 elderly individuals. Specifically, lower actigraphic SE was observed with increasing NV frequency (SE, 80.8\%, $78.8 \%, 79.0 \%, 77.5 \%$, and 77.2\%, respectively) [20]. However, the overall SE was poorer and the detected difference in SE was smaller than those observed in the present study because the study participants were all insomniacs. Therefore, the results of the present study with a large, general, elderly population can be more easily generalized.

The present study also revealed a significant association trend between increased NV frequency and shorter actigraphic SOL. This is inconsistent with the results regarding the association between NV frequency and subjective SOL. Although inconsistency between subjective and actigraphic sleep measures was previously reported [20], a recent study suggested that elderly females with an increased NV frequency had a higher prevalence of excessive daytime sleepiness [26].

The clinical implications of impaired objective sleep quality with increased NV frequency were suggested by a prospective cohort study. In the study, an $11.8 \%$ decrease in actigraphic SE (from the $1^{\text {st }}$ to $3^{\text {rd }}$ quartile) was significantly associated with a $147 \%$ increase in subsequent nursing home placement in 1664 community-dwelling elderly females [27]. The $2.7-5.1 \%$ decrease in actigraphic SE in individuals with two or more NVs, compared with zero $\mathrm{NV}$ in the present study, is predicted to increase subsequent nursing home placement by $33.6-63.5 \%$.

The strengths of the present study include the consideration of information regarding 
endogenous melatonin levels and bedroom light intensity, which could be potential confounding factors. In addition, the study included a large sample of objective sleep measurements in a general elderly population. Previous studies have suggested that endogenous melatonin levels and bedroom light intensity are associated with nocturia and sleep quality [28,29]. Typically, NV with the bedroom light turned on might suppress melatonin secretion and lead to sleep disturbance. However, in the present study, the significant associations identified between NV frequency and both objective and subjective sleep quality were independent of these potential confounding factors.

Some limitations to the present study must be considered. First, it had a cross-sectional design; therefore, the causality of the observed association might be bidirectional [30]. Additional studies with a longitudinal design could accurately assess the magnitude of the effect of NV frequency on objective and subjective sleep quality. The second limitation was the lack of measurements of obstructive sleep apnea (OSA), which might affect both NV frequency and sleep quality [12]. In the present study, predictors of SDB, including OSA, defined by actigraphy were not significantly associated with NV frequency. In addition, the association trends of NV frequency with SE and WASO were independent of probable SDB. However, a potential residual confounding effect of OSA cannot be excluded. The third limitation was that the urination diary did not include information related to fluid intake and caffeine intake, which are associated with NV frequency and sleep quality. Finally, the NV frequency was only measured during a single night; therefore, this parameter might be misclassified in some participants. Nevertheless, 4-months correlation of the NV frequency data was moderate, suggesting that a serious misclassification was infrequent.

In conclusion, the present study demonstrated a quantitative association between NV frequency and objective sleep quality in a large-scale, general, elderly population. 


\section{Acknowledgments}

We would like to thank Sachiko Uemura, Naomi Takenaka, Keiko Nakajima and Nobuhiro Tone for their valuable support during the data collection. This work was supported by grants from the Department of Indoor Environmental Medicine, Nara Medical University; Scientific Research from the Ministry of Education, Culture, Sports, Science and Technology; Mitsui Sumitomo Insurance Welfare Foundation; Meiji Yasuda Life Foundation of Health and Welfare; Osaka Gas Group Welfare Foundation; Japan Diabetes Foundation; Daiwa Securities Health Foundation; the Japan Science and Technology Agency; YKK AP Inc.; Nara Prefecture Health Promotion Foundation; and the Special Collaboration Study from Nara Medical University. 


\section{References}

1. Ford DE, Kamerow DB. Epidemiologic study of sleep disturbances and psychiatric disorders. An opportunity for prevention? JAMA 1989; 262: 1479.

2. Prinz PN, Vitiello MV, Raskind MA et al. Geriatrics: sleep disorders and aging. N Engl J Med 1990; 323: 520.

3. Calem M, Bisla J, Begum A et al. Increased prevalence of insomnia and changes in hypnotics use in England over 15 years: analysis of the 1993, 2000, and 2007 National Psychiatric Morbidity Surveys. Sleep 2012; 35: 377.

4. Walsh JK, Coulouvrat C, Hajak G et al. Nighttime insomnia symptoms and perceived health in the America Insomnia Survey (AIS). Sleep 2011; 34: 997.

5. Eaker, ED, Pinsky J, Castelli WP. Myocardial infarction and coronary death among women: psychosocial predictors from a 20-year follow-up of women in the Framingham Study. Am J Epidemiol 1992; 135: 854.

6. Cricco M, Simonsick EM, Foley DJ. The impact of insomnia on cognitive functioning in older adults. J Am Geriatr Soc 2001; 49: 1185.

7. Yokoyama E, Kaneita Y, Saito Y et al. Association between depression and insomnia subtypes: a longitudinal study on the elderly in Japan. Sleep 2010; 33: 1693.

8. Dew MA, Hoch CC, Buysse DJ et al. Healthy older adults' sleep predicts all-cause mortality at 4 to 19 years of follow-up. Psychosom Med 2003; 65: 63.

9. Yoshimura K, Terada N, Matsui Y et al. Prevalence of and risk factors for nocturia: analysis of a health screening program. Int J Urol 2004; 11: 282.

10. Rembratt A, Norgaard JP, Andersson KE. Nocturia and associated morbidity in a community-dwelling elderly population. BJU Int 2003; 92: 726.

11. Nakagawa H, Niu K, Hozawa A et al. Impact of nocturia on bone fracture and mortality in older individuals: a Japanese longitudinal cohort study. J Urol 2010; 184: 1413. 
12. Parthasarathy S, Fitzgerald M, Goodwin JL et al. Nocturia, sleep-disordered breathing, and cardiovascular morbidity in a community-based cohort. PLoS One 2012; 7: e30969.

13. van Kerrebroeck P, Abrams P, Chaikin D et al. The standardisation of terminology in nocturia: report from the Standardisation Sub-committee of the International Continence Society. Neurourol Urodyn 2002; 21: 179.

14. Asplund R. Nocturia in relation to sleep, somatic diseases and medical treatment in the elderly. BJU Int 2002; 90: 533.

15. Rembratt A, Norgaard JP, Andersson KE. Nocturia and associated morbidity in a community-dwelling elderly population. BJU Int 2003; 92: 726.

16. Coyne KS, Zhou Z, Bhattacharyya SK, et al. The prevalence of nocturia and its effect on health-related quality of life and sleep in a community sample in the USA. BJU Int 2003; 92: 948.

17. Bing MH, Moller LA, Jennum P, et al. Prevalence and bother of nocturia, and causes of sleep interruption in a Danish population of men and women aged 60-80 years. BJU Int 2006; 98: 599.

18. Gopal M, Sammel MD, Pien G, et al. Investigating the associations between nocturia and sleep disorders in perimenopausal women. J Urol 2008; 180: 2063.

19. van den Berg JF, Miedema HM, Tulen JH, et al. Sex differences in subjective and actigraphic sleep measures: a population-based study of elderly persons. Sleep 2009; 32: 1367.

20. Zeitzer JM, Bliwise DL, Hernandez B, et al. Nocturia compounds nocturnal wakefulness in older individuals with insomnia. J Clin Sleep Med 2013; 9: 259.

21. Obayashi K, Saeki K, Iwamoto J, et al. Positive effect of daylight exposure on nocturnal urinary melatonin excretion in the elderly: a cross-sectional analysis of the HEIJO-KYO study. J Clin Endocrinol Metab 2012; 97: 4166. 
22. Philips Respironics Actiware Tutorials: available at http://learnactiware.com/tutorials/ (accessed on August 10, 2014)

23. Mehra R, Stone KL, Ancoli-Israel S, et al. Interpreting wrist actigraphic indices of sleep in epidemiologic studies of the elderly: the Study of Osteoporotic Fractures. Sleep 2008; $31: 1569$.

24. Buysse DJ, Reynolds CF 3rd, Monk TH, et al. The Pittsburgh Sleep Quality Index: a new instrument for psychiatric practice and research. Psychiatry Res 1989; 28:193.

25. Feldstein CA. Nocturia in arterial hypertension: a prevalent, underreported, and sometimes underestimated association. J Am Soc Hypertens 2013; 7: 75.

26. Sacomori C, Cardoso FL, Louzada FM, et al. Excessive daytime sleepiness and nocturia in women. Sleep Med 2014; 15: 677.

27. Spira AP, Covinsky K, Rebok GW, Stone KL, Redline S, Yaffe K. Objectively measured sleep quality and nursing home placement in older women. J Am Geriatr Soc 2012; 60: 1237.

28. Obayashi K, Saeki K, Kurumatani N. Association between melatonin secretion and nocturia in elderly individuals: a cross-Sectional study of the HEIJO-KYO cohort. J Urol 2014; 191: 1816.

29. Obayashi K, Saeki K, Kurumatani N. Association between light exposure at night and insomnia in the general elderly population: The HEIJO-KYO cohort. Chronobiol Int 2014 (in press).

30. Kamperis K, Hagstroem S, Radvanska E, et al. Excess diuresis and natriuresis during acute sleep deprivation in healthy adults. Am J Physiol Renal Physiol 2010; 299: F404. 
Table 1. Basic, Clinical, and Circadian Rhythm Parameters Stratified by NV Frequency

\begin{tabular}{|c|c|c|c|c|c|c|}
\hline \multirow[b]{2}{*}{ Variables } & \multirow[b]{2}{*}{ All } & \multicolumn{4}{|c|}{ NV frequency (time/night) } & \multirow[b]{2}{*}{$P_{\text {trend }}$} \\
\hline & & 0 & 1 & 2 & $\geq 3$ & \\
\hline No. of participants & 1086 & 302 & 462 & 232 & 90 & \\
\hline \multicolumn{7}{|l|}{ Basic and clinical parameters } \\
\hline Age, mean, years & $71.8(7.1)$ & $68.9(6.3)$ & $71.7(6.7)$ & $74.0(6.9)$ & $76.4(7.1)$ & $<0.001$ \\
\hline Gender, number, male & $511(47.1)$ & $118(39.1)$ & $205(44.4)$ & $130(56.0)$ & $58(64.4)$ & $<0.001$ \\
\hline BMI, mean, kg/m² & $23.1(3.1)$ & $23.4(3.2)$ & $23.1(3.0)$ & $22.8(3.0)$ & $23.0(3.1)$ & 0.08 \\
\hline \multicolumn{7}{|l|}{ Antihypertensive drug use } \\
\hline $\mathrm{CCB}$, number & $319(29.4)$ & $61(20.2)$ & $145(31.6)$ & $77(33.2)$ & $36(40.0)$ & $<0.001$ \\
\hline Diuretics, number & $64(5.9)$ & $13(4.3)$ & $30(6.5)$ & $11(4.7)$ & $10(11.1)$ & 0.10 \\
\hline $\mathrm{BB}$, number & $40(3.7)$ & $6(2.0)$ & $18(3.9)$ & $14(6.0)$ & $2(2.2)$ & 0.15 \\
\hline $\mathrm{BPH}$, number & $110(21.7)$ & $14(11.9)$ & $36(17.6)$ & $37(28.7)$ & $23(40.4)$ & $<0.001$ \\
\hline Sleep medication, number & $115(10.6)$ & $22(7.3)$ & $46(10.0)$ & $40(17.3)$ & $7(7.8)$ & 0.026 \\
\hline Diabetes, number & $128(11.9)$ & $22(7.3)$ & $65(14.2)$ & $29(12.7)$ & $12(13.6)$ & 0.051 \\
\hline eGFR, mean, $\mathrm{mL} / \mathrm{min} / 1.73 \mathrm{~m}^{2}$ & $72.4(15.2)$ & $73.9(14.4)$ & $72.8(14.7)$ & $70.8(16.3)$ & $70.0(16.8)$ & 0.006 \\
\hline \multicolumn{7}{|l|}{ Circadian rhythm parameters } \\
\hline Bedtime, mean, clock time & $22: 29(1: 10)$ & $22: 54(1: 08)$ & $22: 32(1: 05)$ & $22: 05(1: 09)$ & $21: 50(1: 06)$ & $<0.001$ \\
\hline Rising time, mean, clock time & $6: 46(0: 56)$ & $6: 36(0: 56)$ & $6: 48(0: 55)$ & $6: 50(0: 57)$ & $6: 58(0: 52)$ & $<0.001$ \\
\hline Daytime physical activity, mean, counts/min & $296.9(101.6)$ & $315.2(101.8)$ & $297.8(98.2)$ & $280.2(106.4)$ & $273.7(95.4)$ & $<0.001$ \\
\hline UME, mean, $\log \mu \mathrm{g}$ & $1.9(0.7)$ & $1.9(0.7)$ & $1.9(0.7)$ & $1.8(0.7)$ & $1.8(0.7)$ & 0.07 \\
\hline Bedroom light intensity, median, lux & $0.7(0.1,3.3)$ & $0.6(0.1,3.3)$ & $0.7(0.1,3.2)$ & $0.7(0.2,2.9)$ & $2.0(0.1,7.7)$ & 0.13 \\
\hline
\end{tabular}

Data are expressed as mean (SD), number (\%), or median (interquartile range). NV, nocturnal void; BMI, body mass index; CCB, calcium channel blocker; $\mathrm{BB}$, beta blocker; $\mathrm{BPH}$, benign prostatic hyperplasia; eGFR, estimated glomerular filtration rate; UME, urinary 6-sulfatoxymelatonin excretion. 
Table 2. Comparisons of Objective Parameters of Sleep Quality Stratified by NV Frequency

\begin{tabular}{|c|c|c|c|c|c|c|}
\hline \multirow{2}{*}{ Variables } & & \multicolumn{4}{|c|}{ NV frequency (time/night) } & \multirow[b]{2}{*}{$P_{\text {trend }}$} \\
\hline & & 0 & 1 & 2 & $\geq 3$ & \\
\hline No. of participants & & 302 & 462 & 232 & 90 & \\
\hline \multicolumn{7}{|l|}{ Unadjusted } \\
\hline $\mathrm{SE}, \%$ & mean (SD) & $86.7(6.3)$ & $84.9(7.4)$ & $83.3(8.2)$ & $80.2(8.9)$ & $<0.001$ \\
\hline WASO, min & mean (SD) & $37.8(22.8)$ & $48.7(26.4)$ & $57.2(32.0)$ & $73.9(32.2)$ & $<0.001$ \\
\hline $\mathrm{SOL}, \min$ & median (interquartile range) & $17.8(9.5,33.0)$ & $19.5(10.0,39.5)$ & $17.8(8.5,37.0)$ & $19.8(10.3,41.5)$ & 0.50 \\
\hline $\mathrm{SOL}, \log \min$ & mean (SD) & $2.9(0.9)$ & $3.0(0.9)$ & $2.9(1.0)$ & $3.0(1.1)$ & 0.39 \\
\hline $\mathrm{TST}, \min$ & mean (SD) & $411.6(69.8)$ & $441.9(72.6)$ & $463.4(81.5)$ & $480.5(89.7)$ & $<0.001$ \\
\hline \multicolumn{7}{|l|}{ Adjusted $\dagger$} \\
\hline \multirow[t]{3}{*}{$\mathrm{SE}, \%$} & mean $(95 \% \mathrm{CI})$ & $86.3(85.5,87.2)$ & $84.8(84.2,85.5)$ & $83.6(82.6,84.6)$ & $81.2(79.6,82.7)$ & $<0.001$ \\
\hline & difference $(95 \% \mathrm{CI})$ & reference & $1.5(0.4,2.6)$ & $2.7(1.4,4.1)$ & $5.1(3.3,7.0)$ & \\
\hline & $P$ value & & 0.006 & $<0.001$ & $<0.001$ & \\
\hline \multirow[t]{3}{*}{ WASO, min } & mean $(95 \% \mathrm{CI})$ & $42.6(39.6,45.6)$ & $48.9(46.6,51.3)$ & $53.6(50.3,57.0)$ & $66.1(60.7,71.5)$ & $<0.001$ \\
\hline & difference $(95 \% \mathrm{CI})$ & reference & $6.3(2.5,10.1)$ & $11.0(6.4,15.7)$ & $23.5(17.1,29.9)$ & \\
\hline & $P$ value & & 0.001 & $<0.001$ & $<0.001$ & \\
\hline \multirow[t]{3}{*}{ SOL, log min } & mean $(95 \% \mathrm{CI})$ & $3.0(2.9,3.1)$ & $3.0(2.9,3.1)$ & $2.8(2.7,2.9)$ & $2.8(2.6,3.0)$ & 0.018 \\
\hline & difference $(95 \% \mathrm{CI})$ & reference & $-0.04(-0.2,0.1)$ & $-0.2(-0.4,-0.03)$ & $-0.2(-0.4,0.1)$ & \\
\hline & $P$ value & & 0.60 & 0.020 & 0.12 & \\
\hline \multirow[t]{3}{*}{$\mathrm{TST}, \min$} & mean $(95 \% \mathrm{CI})$ & $440.6(435.3,446.0)$ & $442.3(438.2,446.5)$ & $440.1(434.1,446.1)$ & $440.8(431.1,450.5)$ & 0.91 \\
\hline & difference $(95 \% \mathrm{CI})$ & reference & $1.7(-5.1,8.5)$ & $-0.5(-8.8,7.8)$ & $0.2(-11.3,11.6)$ & \\
\hline & $P$ value & & 0.63 & 0.90 & 0.98 & \\
\hline
\end{tabular}

NV, nocturnal void; SE, sleep efficiency; SOL, sleep-onset latency; WASO, wake after sleep-onset; TST, total sleep time; SD, standard deviation; CI, confidence interval; BMI, body mass index; CCB, calcium channel blocker; eGFR, estimated glomerular filtration rate; UME, urinary 6-sulfatoxymelatonin excretion.

$\dagger$ Adjusted for age (per 5 years), gender, BMI, CCB use, sleep medication use, diabetes, eGFR (per $10 \mathrm{~mL} / \mathrm{min} / 1.73 \mathrm{~m}^{2}$ ), bedtime (per 30 min delay), rising time (per 30 min delay), daytime physical activity (per 100 counts/min), UME, and bedroom light intensity (per quartile). 
Table 3. Comparisons of Subjective Parameters of Sleep Quality Stratified by NV Frequency

\begin{tabular}{|c|c|c|c|c|c|}
\hline \multirow[b]{2}{*}{ Variables } & \multicolumn{4}{|c|}{ NV frequency (time/night) } & \multirow[b]{2}{*}{$P_{\text {trend }}$} \\
\hline & 0 & 1 & 2 & $\geq 3$ & \\
\hline No. of participants & 302 & 462 & 232 & 90 & \\
\hline \multicolumn{6}{|l|}{ Unadjusted } \\
\hline Global PSQI score, mean (SD) & $4.41(2.97)$ & $4.87(3.19)$ & $5.37(3.55)$ & $5.73(3.62)$ & $<0.001$ \\
\hline \multicolumn{6}{|l|}{ PSQI component, mean (SD) } \\
\hline Sleep quality & $0.87(0.63)$ & $0.97(0.61)$ & $1.08(0.66)$ & $1.11(0.67)$ & $<0.001$ \\
\hline Sleep latency & $0.78(0.89)$ & $0.98(0.91)$ & $1.04(0.90)$ & $1.33(1.02)$ & $<0.001$ \\
\hline Sleep duration & $1.13(0.90)$ & $0.92(0.90)$ & $0.81(0.91)$ & $0.77(1.00)$ & $<0.001$ \\
\hline Sleep efficiency & $0.38(0.79)$ & $0.48(0.84)$ & $0.61(0.94)$ & $0.81(1.10)$ & $<0.001$ \\
\hline Sleep disturbances & $0.69(0.52)$ & $0.86(0.52)$ & $0.94(0.55)$ & $0.98(0.62)$ & $<0.001$ \\
\hline Sleep medication use & $0.31(0.86)$ & $0.38(0.92)$ & $0.58(1.13)$ & $0.37(0.97)$ & 0.031 \\
\hline Daytime dysfunction & $0.25(0.54)$ & $0.29(0.56)$ & $0.32(0.59)$ & $0.38(0.65)$ & 0.039 \\
\hline \multicolumn{6}{|l|}{ Adjusted $\dagger$} \\
\hline Global PSQI score, mean $(95 \% \mathrm{CI})$ & $4.61(4.23,4.99)$ & $4.87(4.58,5.16)$ & $5.22(4.80,5.64)$ & $5.48(4.80,6.17)$ & 0.012 \\
\hline difference $(95 \% \mathrm{CI})$ & reference & $0.26(-0.22,0.74)$ & $0.61(0.03,1.20)$ & $0.87(0.07,1.68)$ & \\
\hline$P$ value & & 0.28 & 0.041 & 0.034 & \\
\hline \multicolumn{6}{|l|}{ PSQI component, mean $(95 \% \mathrm{CI})$} \\
\hline Sleep quality & $0.88(0.80,0.95)$ & $0.97(0.91,1.02)$ & $1.08(0.99,1.16)$ & $1.12(0.99,1.26)$ & $<0.001$ \\
\hline Sleep latency & $0.89(0.79,1.00)$ & $0.98(0.90,1.06)$ & $0.95(0.84,1.07)$ & $1.18(0.99,1.36)$ & 0.045 \\
\hline Sleep duration & $0.99(0.89,1.09)$ & $0.92(0.84,1.00)$ & $0.92(0.80,1.03)$ & $0.96(0.77,1.15)$ & 0.57 \\
\hline Sleep efficiency & $0.51(0.41,0.61)$ & $0.49(0.41,0.61)$ & $0.50(0.39,0.61)$ & $0.63(0.45,0.81)$ & 0.47 \\
\hline Sleep disturbances & $0.72(0.66,0.78)$ & $0.86(0.81,0.91)$ & $0.92(0.85,0.99)$ & $0.94(0.83,1.06)$ & $<0.001$ \\
\hline Sleep medication use & $0.40(0.29,0.51)$ & $0.38(0.29,0.46)$ & $0.52(0.40,0.64)$ & $0.26(0.06,0.46)$ & 0.94 \\
\hline Daytime dysfunction & $0.23(0.16,0.29)$ & $0.29(0.23,0.34)$ & $0.34(0.27,0.42)$ & $0.41(0.29,0.53)$ & 0.005 \\
\hline
\end{tabular}

NV, nocturnal void; PSQI, Pittsburgh Sleep Quality Index; CI, confidence interval; SD, standard deviation; BMI, body mass index; CCB, calcium channel blocker; eGFR, estimated glomerular filtration rate; UME, urinary 6-sulfatoxymelatonin excretion.

$\uparrow$ Adjusted for age (per 5 years), gender, BMI, CCB use, diabetes, eGFR (per $10 \mathrm{~mL} / \mathrm{min} / 1.73 \mathrm{~m}^{2}$ ), bedtime (per 30 min delay), rising time (per 30 min delay), daytime physical activity (per 100 counts/min), UME, and bedroom light intensity (per quartile). 
Table 4. Comparisons of Subjective and Objective Sleep Quality between NV $\geq 2$ and $<2$ Times

\begin{tabular}{lccc}
\hline & \multicolumn{2}{c}{ NV frequency (time/night) } & \\
\cline { 2 - 3 } & 764 & $\geq 2$ & \\
\hline No. of participants & & 322 & \\
Objective sleep quality (mean, 95\%CI) $\dagger$ & $85.4(84.8,85.9)$ & $83.0(82.2,83.9)$ & $<0.001$ \\
SE, \% & $46.7(44.8,48.5)$ & $56.6(53.7,59.6)$ & $<0.001$ \\
WASO, min & $3.0(2.9,3.1)$ & $2.8(2.7,2.9)$ & 0.009 \\
SOL, log min & $441.7(438.4445 .0)$ & $440.2(435.0,445.4)$ & 0.64 \\
TST, min & & & \\
Sujective sleep quality (mean, 95\%CI) & & & \\
Global PSQI score & $4.77(4.54,5.00)$ & $5.27(4.91,5.64)$ & 0.028 \\
\hline
\end{tabular}

NV, nocturnal void; CI, confidence interval; SE, sleep efficiency; SOL, sleep-onset latency; WASO, wake after sleep-onset; TST, total sleep time; PSQI, Pittsburgh Sleep Quality Index; BMI, body mass index; $\mathrm{CCB}$, calcium channel blocker; eGFR, estimated glomerular filtration rate; UME, urinary 6sulfatoxymelatonin excretion.

$\dagger$ Adjusted for age (per 5 years), gender, BMI, CCB use, sleep medication use, diabetes, eGFR (per 10 $\mathrm{mL} / \mathrm{min} / 1.73 \mathrm{~m} 2$ ), bedtime (per $30 \mathrm{~min}$ delay), rising time (per 30 min delay), daytime physical activity (per 100 counts/min), UME, and bedroom light intensity (per quartile).

$\ddagger$ Adjusted for age (per 5 years), gender, BMI, CCB use, diabetes, eGFR (per $10 \mathrm{~mL} / \mathrm{min} / 1.73 \mathrm{~m} 2$ ), bedtime (per 30 min delay), rising time (per 30 min delay), daytime physical activity (per 100 counts/min), UME, and bedroom light intensity (per quartile). 